\title{
OSTROWSKI TYPE INEQUALITIES FOR CONVEX FUNCTIONS
}

\author{
M. EMIN ÖZDEMIR, HAVVA KAVURMACI AND MERVE AVCI
}

Abstract. In this paper, we obtain Ostrowski type inequalities for convex functions.

\section{Introduction}

A function $f: I \rightarrow \mathbb{R}$ is said to be convex function on $I$ if the inequality

$$
f(\alpha x+(1-\alpha) y) \leq \alpha f(x)+(1-\alpha) f(y)
$$

holds for all $x, y \in I$ and $\alpha \in[0,1]$.

Let $f: I \subset[0, \infty) \rightarrow \mathbb{R}$ be a differentiable mapping on $I^{\circ}$, the interior of the interval $I$, such that $f^{\prime} \in L[a, b]$ where $a, b \in I$ with $a<b$. If $\left|f^{\prime}\right| \leq M$, then the following inequality holds (See [1])

$$
\left|f(x)-\frac{1}{b-a} \int_{a}^{b} f(u) d u\right| \leq \frac{M}{b-a}\left[\frac{(x-a)^{2}+(b-x)^{2}}{2}\right] .
$$

This inequality is well known in the literature as the Ostrowski inequality. For some results which generalize, improve and extend the inequality (1.1) see [1]-[14].

\section{Inequalities for Convex Functions}

We use the following Lemma to obtain our main results.

Lemma 1. Let $I \subset \mathbb{R}$ be an open interval and $f: I \subset \mathbb{R} \rightarrow \mathbb{R}$ be a differentiable function on $I^{\circ}$ where $a, b \in I$ with $a<b$. If $f^{\prime} \in L_{1}[a, b]$ for $\lambda \in[0,1]$ and $x \in[a, b]$, the following equality holds

$$
\begin{aligned}
& \frac{1}{b-a} \int_{a}^{b} f(u) d u-\frac{(b-x)[(1-\lambda) f(x)+\lambda f(b)]+(x-a)[(1-\lambda) f(x)+\lambda f(a)]}{b-a} \\
& \quad=(b-a) \int_{0}^{1} h(t, \lambda) f^{\prime}(t a+(1-t) b) d t
\end{aligned}
$$

Received April 10, 2012, accepted November 7, 2013.

2010 Mathematics Subject Classification. Primary 26D15; Secondary 26A51.

Key words and phrases. Ostrowski inequality, convex functions, Hölder inequality, power-mean inequality.

Corresponding author: Havva Kavurmacl. 
where

$$
h(t, \lambda)= \begin{cases}t-\lambda\left(\frac{b-x}{b-a}\right), & t \in\left[0, \frac{b-x}{b-a}\right) \\ t-1+\lambda\left(\frac{x-a}{b-a}\right), & t \in\left(\frac{b-x}{b-a}, 1\right] .\end{cases}
$$

Proof. We note that

$$
\begin{aligned}
K & =\int_{0}^{1} h(t, \lambda) f^{\prime}(t a+(1-t) b) d t \\
& =\int_{0}^{\frac{b-x}{b-a}}\left[t-\lambda\left(\frac{b-x}{b-a}\right)\right] f^{\prime}(t a+(1-t) b) d t+\int_{\frac{b-x}{b-a}}^{1}\left[t-1+\lambda\left(\frac{x-a}{b-a}\right)\right] f^{\prime}(t a+(1-t) b) d t .
\end{aligned}
$$

Integrating by parts we have

$$
\begin{aligned}
K= & {\left.\left[t-\lambda\left(\frac{b-x}{b-a}\right)\right] \frac{f(t a+(1-t) b)}{a-b}\right|_{0} ^{\frac{b-x}{b-a}}-\int_{0}^{\frac{b-x}{b-a}} \frac{f(t a+(1-t) b)}{a-b} d t } \\
& +\left.\left[t-1+\lambda\left(\frac{x-a}{b-a}\right)\right] \frac{f(t a+(1-t) b)}{a-b}\right|_{\frac{b-x}{b-a}} ^{1}-\int_{\frac{b-x}{b-a}}^{1} \frac{f(t a+(1-t) b)}{a-b} d t \\
= & \frac{1}{(b-a)^{2}} \int_{a}^{b} f(u) d u-\frac{(b-x)[(1-\lambda) f(x)+\lambda f(b)]+(x-a)[(1-\lambda) f(x)+\lambda f(a)]}{(b-a)^{2}} .
\end{aligned}
$$

If we multiply the resulting equality with $(b-a)$, we complete the proof.

Theorem 1. Let $I \subset \mathbb{R}$ be an open interval and $f: I \subset \mathbb{R} \rightarrow \mathbb{R}$ be a differentiable function where $a, b \in I$ with $a<b$. If $\left|f^{\prime}\right|^{q}$ is convex function for $\lambda \in[0,1], x \in[a, b]$ and $q \in[1, \infty)$, then the following inequality holds:

$$
\begin{aligned}
& \left|\frac{1}{b-a} \int_{a}^{b} f(u) d u-\frac{(b-x)[(1-\lambda) f(x)+\lambda f(b)]+(x-a)[(1-\lambda) f(x)+\lambda f(a)]}{b-a}\right| \\
& \leq(b-a)\left(\frac{\left(2 \lambda^{2}-2 \lambda+1\right)}{2}\right)^{\frac{(a-1)}{q}}\left\{\left[\left(\frac{2 \lambda^{3}-3 \lambda+2}{6}\right)\left(\frac{b-x}{b-a}\right)^{2 q+1}\left|f^{\prime}(a)\right|^{q}\right.\right. \\
& \left.\quad+\left(\frac{\left[6 \lambda^{2}-6 \lambda+3\right]-\left[2 \lambda^{3}-3 \lambda+2\right]\left(\frac{b-x}{b-a}\right)}{6}\right)\left(\frac{b-x}{b-a}\right)^{2 q}\left|f^{\prime}(b)\right|^{q}\right]^{\frac{1}{q}} \\
& \quad+\left[\left(\frac{\left[6 \lambda^{2}-6 \lambda+3\right]-\left[2 \lambda^{3}-3 \lambda+2\right]\left(\frac{x-a}{b-a}\right)}{6}\right)\left(\frac{x-a}{b-a}\right)^{2 q}\left|f^{\prime}(a)\right|^{q}\right. \\
& \left.\left.\quad+\left(\frac{2 \lambda^{3}-3 \lambda+2}{6}\right)\left(\frac{x-a}{b-a}\right)^{2 q+1}\left|f^{\prime}(b)\right|^{q}\right]^{\frac{1}{q}}\right\} .
\end{aligned}
$$

Proof. Suppose that $q=1$. From Lemma 1 and using the properties of modulus, we have

$$
\left|\frac{1}{b-a} \int_{a}^{b} f(u) d u-\frac{(b-x)[(1-\lambda) f(x)+\lambda f(b)]+(x-a)[(1-\lambda) f(x)+\lambda f(a)]}{b-a}\right|
$$




$$
\begin{aligned}
\leq & (b-a)\left\{\int_{0}^{\frac{b-x}{b-a}}\left|t-\lambda\left(\frac{b-x}{b-a}\right)\right|\left|f^{\prime}(t a+(1-t) b)\right| d t\right. \\
& \left.+\int_{\frac{b-x}{b-a}}^{1}\left|t-1+\lambda\left(\frac{x-a}{b-a}\right)\right|\left|f^{\prime}(t a+(1-t) b)\right| d t\right\} .
\end{aligned}
$$

Since $\left|f^{\prime}\right|$ is convex function on $[a, b]$, we can write

$$
\left|f^{\prime}(t a+(1-t) b)\right| \leq t\left|f^{\prime}(a)\right|+(1-t)\left|f^{\prime}(b)\right| .
$$

Hence,

$$
\begin{aligned}
& \mid \frac{1}{b-a} \int_{a}^{b} f(u) d u-\frac{(b-x)[(1-\lambda) f(x)+\lambda f(b)]+(x-a)[(1-\lambda) f(x)+\lambda f(a)]}{b-a} \mid \\
& \leq(b-a)\left\{\int_{0}^{\frac{b-x}{b-a}}\left|t-\lambda\left(\frac{b-x}{b-a}\right)\right|\left[t\left|f^{\prime}(a)\right|+(1-t)\left|f^{\prime}(b)\right|\right] d t\right. \\
&\left.+\int_{\frac{b-x}{b-a}}^{1}\left|t-1+\lambda\left(\frac{x-a}{b-a}\right)\right|\left[t\left|f^{\prime}(a)\right|+(1-t)\left|f^{\prime}(b)\right|\right] d t\right\} \\
&=(b-a)\left\{\left|f^{\prime}(a)\right|\left[\int_{0}^{\frac{b-x}{b-a}}\left|t-\lambda\left(\frac{b-x}{b-a}\right)\right| t d t+\int_{\frac{b-x}{b-a}}^{1}\left|t-1+\lambda\left(\frac{x-a}{b-a}\right)\right| t d t\right]\right. \\
&\left.\quad+\left|f^{\prime}(b)\right|\left[\int_{0}^{\frac{b-x}{b-a}}\left|t-\lambda\left(\frac{b-x}{b-a}\right)\right|(1-t) d t+\int_{\frac{b-x}{b-a}}^{1}\left|t-1+\lambda\left(\frac{x-a}{b-a}\right)\right|(1-t) d t\right]\right\} .
\end{aligned}
$$

To complete the proof for this case we used the fact that

$$
\begin{aligned}
\int_{0}^{\frac{b-x}{b-a}}\left|t-\lambda\left(\frac{b-x}{b-a}\right)\right| t d t & =\left(\frac{2 \lambda^{3}-3 \lambda+2}{6}\right)\left(\frac{b-x}{b-a}\right)^{3}, \\
\int_{\frac{b-x}{b-a}}^{1}\left|t-1+\lambda\left(\frac{x-a}{b-a}\right)\right| t d t & =\left(\frac{\left[6 \lambda^{2}-6 \lambda+3\right]-\left[2 \lambda^{3}-3 \lambda+2\right]\left(\frac{x-a}{b-a}\right)}{6}\right)\left(\frac{x-a}{b-a}\right)^{2}, \\
\int_{0}^{\frac{b-x}{b-a}}\left|t-\lambda\left(\frac{b-x}{b-a}\right)\right|(1-t) d t & =\left(\frac{\left[6 \lambda^{2}-6 \lambda+3\right]-\left[2 \lambda^{3}-3 \lambda+2\right]\left(\frac{b-x}{b-a}\right)}{6}\right)\left(\frac{b-x}{b-a}\right)^{2}
\end{aligned}
$$

and

$$
\int_{\frac{b-x}{b-a}}^{1}\left|t-1+\lambda\left(\frac{x-a}{b-a}\right)\right|(1-t) d t=\left(\frac{2 \lambda^{3}-3 \lambda+2}{6}\right)\left(\frac{x-a}{b-a}\right)^{3}
$$

in (2.1).

Now, suppose that $q>1$. From Lemma 1, using Hölder inequality and convexity of $\left|f^{\prime}\right|^{q}$, we have

$$
\left|\frac{1}{b-a} \int_{a}^{b} f(u) d u-\frac{(b-x)[(1-\lambda) f(x)+\lambda f(b)]+(x-a)[(1-\lambda) f(x)+\lambda f(a)]}{b-a}\right|
$$




$$
\begin{aligned}
\leq & (b-a)\left\{\int_{0}^{\frac{b-x}{b-a}}\left|t-\lambda\left(\frac{b-x}{b-a}\right)\right|^{\frac{q-1}{q}}\left|t-\lambda\left(\frac{b-x}{b-a}\right)\right|^{\frac{1}{q}}\left|f^{\prime}(t a+(1-t) b)\right| d t\right. \\
& \left.+\int_{\frac{b-x}{b-a}}^{1}\left|t-1+\lambda\left(\frac{x-a}{b-a}\right)\right|^{\frac{q-1}{q}}\left|t-1+\lambda\left(\frac{x-a}{b-a}\right)\right|^{\frac{1}{q}}\left|f^{\prime}(t a+(1-t) b)\right| d t\right\} \\
\leq & (b-a)\left\{\left(\int_{0}^{\frac{b-x}{b-a}}\left|t-\lambda\left(\frac{b-x}{b-a}\right)\right| d t\right)^{\frac{q-1}{q}}\right. \\
& \times\left(\int_{0}^{\frac{b-x}{b-a}}\left|t-\lambda\left(\frac{b-x}{b-a}\right)\right|\left|f^{\prime}(t a+(1-t) b)\right|^{q} d t\right)^{\frac{1}{q}} \\
& +\left(\int_{\frac{b-x}{b-a}}^{1}\left|t-1+\lambda\left(\frac{x-a}{b-a}\right)\right| d t\right)^{\frac{q-1}{q}} \\
& \left.\times\left(\int_{\frac{b-x}{b-a}}^{1}\left|t-1+\lambda\left(\frac{x-a}{b-a}\right)\right|\left|f^{\prime}(t a+(1-t) b)\right|^{q} d t\right)^{\frac{1}{q}}\right\} \\
\leq & (b-a)\left\{\left(\int_{0}^{\frac{b-x}{b-a}}\left|t-\lambda\left(\frac{b-x}{b-a}\right)\right| d t\right)^{\frac{q-1}{q}}\right. \\
& \left.\times\left(\int_{\frac{b-x}{b-a}}^{1}\left|t-1+\lambda\left(\frac{x-a}{b-a}\right)\right|\left[t\left|f^{\prime}(a)\right|^{q}+(1-t)\left|f^{\prime}(b)\right|^{q}\right] d t\right)^{\frac{1}{q}}\right\} . \\
& \times\left(\int_{0}^{\frac{b-x}{b-a}}\left|t-\lambda\left(\frac{b-x}{b-a}\right)\right|\left[t\left|f^{\prime}(a)\right|^{q}+(1-t)\left|f^{\prime}(b)\right|^{q}\right] d t\right)^{\frac{1}{q}}
\end{aligned}
$$

If we use the inequalities in (2.2)-(2.5) and the following two inequalities in the last inequality, we obtain the required result.

We note that

$$
\int_{0}^{\frac{b-x}{b-a}}\left|t-\lambda\left(\frac{b-x}{b-a}\right)\right| d t=\left(\frac{2 \lambda^{2}-2 \lambda+1}{2}\right)\left(\frac{b-x}{b-a}\right)^{2}
$$

and

$$
\int_{\frac{b-x}{b-a}}^{1}\left|t-1+\lambda\left(\frac{x-a}{b-a}\right)\right| d t=\left(\frac{2 \lambda^{2}-2 \lambda+1}{2}\right)\left(\frac{x-a}{b-a}\right)^{2} .
$$

Theorem 2. Let $I \subset \mathbb{R}$ be an open interval and $f: I \subset \mathbb{R} \rightarrow \mathbb{R}$ be a differentiable function where $a, b \in I$ with $a<b$. If $\left|f^{\prime}\right|^{q}$ is convex function for $\lambda \in[0,1], x \in[a, b]$ and $q \in(1, \infty)$, then the following inequality holds:

$$
\left|\frac{1}{b-a} \int_{a}^{b} f(u) d u-\frac{(b-x)[(1-\lambda) f(x)+\lambda f(b)]+(x-a)[(1-\lambda) f(x)+\lambda f(a)]}{b-a}\right|
$$




$$
\begin{aligned}
\leq & (b-a)\left(\frac{\lambda^{p+1}+(1-\lambda)^{p+1}}{p+1}\right)^{\frac{1}{p}} \\
& \times\left\{\left(\frac{b-x}{b-a}\right)^{\frac{p+1}{p}}\left(\frac{\left(\frac{b-x}{b-a}\right)^{2}}{2}\left|f^{\prime}(a)\right|^{q}+\left(\frac{1}{2}-\frac{\left(\frac{x-a}{b-a}\right)^{2}}{2}\right)\left|f^{\prime}(b)\right|^{q}\right)^{\frac{1}{q}}\right. \\
& \left.+\left(\frac{x-a}{b-a}\right)^{\frac{p+1}{p}}\left(\left(\frac{1}{2}-\frac{\left(\frac{b-x}{b-a}\right)^{2}}{2}\right)\left|f^{\prime}(a)\right|^{q}+\frac{\left(\frac{x-a}{b-a}\right)^{2}}{2}\left|f^{\prime}(b)\right|^{q}\right)^{\frac{1}{q}}\right\} .
\end{aligned}
$$

Proof. From Lemma 1, using the properties of modulus and Hölder inequality, we have

$$
\begin{aligned}
& \left|\frac{1}{b-a} \int_{a}^{b} f(u) d u-\frac{(b-x)[(1-\lambda) f(x)+\lambda f(b)]+(x-a)[(1-\lambda) f(x)+\lambda f(a)]}{b-a}\right| \\
& \leq(b-a)\left\{\left(\int_{0}^{\frac{b-x}{b-a}}\left|t-\lambda\left(\frac{b-x}{b-a}\right)\right|^{p} d t\right)^{\frac{1}{p}}\left(\int_{0}^{\frac{b-x}{b-a}}\left|f^{\prime}(t a+(1-t) b)\right|^{q} d t\right)^{\frac{1}{q}}\right. \\
& \left.\quad+\left(\int_{\frac{b-x}{b-a}}^{1}\left|t-1+\lambda\left(\frac{x-a}{b-a}\right)\right|^{p} d t\right)^{\frac{1}{p}}\left(\int_{\frac{b-x}{b-a}}^{1}\left|f^{\prime}(t a+(1-t) b)\right|^{q} d t\right)^{\frac{1}{q}}\right\} .
\end{aligned}
$$

Since $\left|f^{\prime}\right|^{q}$ is convex function we can write

$$
\int_{0}^{\frac{b-x}{b-a}}\left|f^{\prime}(t a+(1-t) b)\right|^{q} d t \leq \frac{\left(\frac{b-x}{b-a}\right)^{2}}{2}\left|f^{\prime}(a)\right|^{q}+\left(\frac{1}{2}-\frac{\left(\frac{x-a}{b-a}\right)^{2}}{2}\right)\left|f^{\prime}(b)\right|^{q}
$$

and

$$
\int_{\frac{b-x}{b-a}}^{1}\left|f^{\prime}(t a+(1-t) b)\right|^{q} d t=\left(\frac{1}{2}-\frac{\left(\frac{b-x}{b-a}\right)^{2}}{2}\right)\left|f^{\prime}(a)\right|^{q}+\frac{\left(\frac{x-a}{b-a}\right)^{2}}{2}\left|f^{\prime}(b)\right|^{q} .
$$

By a simple calculation, we also have

$$
\int_{0}^{\frac{b-x}{b-a}}\left|t-\lambda\left(\frac{b-x}{b-a}\right)\right|^{p} d t=\left(\frac{\lambda^{p+1}+(1-\lambda)^{p+1}}{p+1}\right)\left(\frac{b-x}{b-a}\right)^{p+1}
$$

and

$$
\int_{\frac{b-x}{b-a}}^{1}\left|t-1+\lambda\left(\frac{x-a}{b-a}\right)\right|^{p} d t=\left(\frac{\lambda^{p+1}+(1-\lambda)^{p+1}}{p+1}\right)\left(\frac{x-a}{b-a}\right)^{p+1} .
$$

By using the inequalities in (2.7)-(2.10) in the inequality (2.6), we obtained the required result.

\section{References}

[1] A. Ostrowski, Uber die Absolutabweichung einer differentienbaren Funktionen von ihren Integralmittelwert. Comment. Math. Hel, 10 (1938), 226-227. 
[2] S. S. Dragomir and A. Sofo, Ostrowski type inequalities for functions whose derivatives are convex, RGMIA Res. Rep. Coll., 5 (2002) Supplement, Article 30. Online: http://rgmia.vu.edu.au/v5(E).html

[3] S.S. Dragomir, Some companions of Ostrowski's inequality for absolutely continuous functions and applications, Bull. Korean Math. Soc., 42(2005), 213-230.

[4] S. S. Dragomir and T. M. Rassias, Ostrowski type inequalities and applications in numerical integration, Boston: Kluwer Academic, 404 pp, Melbourne-Athens, (2002).

[5] S. S. Dragomir, The Ostrowski's integral inequality for Lipschitzian mappings and applications, Computers and Mathematics with Applications, 38 (1997), 33-37.

[6] S. S. Dragomir and S. Wang, Applications of Ostrowski's inequality to the estimation of error bounds for some special means and for some numerical quadrature rules, Appl. Math. Lett., 11 (1998), 105-109.

[7] S. S. Dragomir and S. Wang, An inequality of Ostrowski-Grüss' type and its applications to the estimation of error bounds for some special means and for some numerical quadrature rules, Computers Math. Applic., 33(11) (1997), 15-20.

[8] N. S. Barnett and S. S. Dragomir, Applications of Ostrowski's version of the Grüss inequality for trapezoid type rules, Tamkang Journal of Mathematics, 37(2006), 163-173.

[9] N. S. Barnett and S. S. Dragomir, An Ostrowski type inequality for double integrals and applications for cubature formulae, Soochow Journal of Mathematics, 27(2001), 1-10.

[10] M. W. Alomari, M. E. Özdemir and H. Kavurmacı, On Companion of Ostrowski Inequality for Mappings Whose First Derivatives Absolute Value Are Convex With Applications, Miskolc Mathematical Notes, 13 (2012), 233248.

[11] M. E. Özdemir, H. Kavurmacı and E. Set, Ostrowski's Type Inequalities for $(\alpha, m)$-Convex Functions, Kyungpook Math. J., 50(2010), 371-378.

[12] M. Alomari, M. Darus, S. S. Dragomir and P. Cerone, Ostrowski type inequalities for functions whose derivatives are convex in the second sense, Applied Mathematics Letters, 23 (2010), 1071-1076.

[13] M. Emin Özdemir, Ahmet Ocak Akdemir and Erhan Set, On the Ostrowski-Grüss type inequality for twice differentiable functions, Hacettepe Journal of Mathematics and Statistics, 41(2012), 651-655.

[14] M. Emin Özdemir, Ahmet Ocak Akdemir and Erhan Set, A new Ostrowski-type inequality for double integrals, Journal of Inequalities and Special Functions, ISSN: 2217-4303, URL: http: //www . ilirias . com, 2(2011), 27-34.

Department Of Mathematics, K.K. Education Faculty, Atatürk University, 25240, Campus, Erzurum, Turkey.

E-mail: emos@atauni.edu.tr

Department of Mathematics, Education Faculty, Önalan Yüzüncü Yıl University, 65080, Van, Turkey.

E-mail: hkavurmaci@yyu.edu.tr

Department Of Mathematics, Faculty Of Science And Art, Adıyaman University, 02040, Adıyaman, Turkey.

E-mail: mavci@posta.adiyaman.edu.tr 